\title{
Two-dimensional forward simulation method for ground penetrating radar detection
}

\author{
Yao Li, Bin Liu, Shucai Li, Fengkai Zhang \\ Geotechnical and Structural Engineering Research Center \\ Shandong University \\ Jinan, China
}

\begin{abstract}
In recent years, tunnels and underground engineering are developing rapidly, and meanwhile the topography, complex geological conditions making interpretation difficult in case of ground penetrating radar. To solve this problem, we introduce a two-dimensional forward simulation method for ground penetrating radar detection based on finitedifference time-domain method, which is proved to be effective through a numerical example: the position and shape of the adverse geological body can be inferred by analyzing the reflected signal from the finite-difference time-domain simulation results; and the relationship between the relative dielectric constants of the adverse geological body and the surrounding medium can be found by analyzing the phase information of the reflected signal.
\end{abstract}

Keywords-forward simulation; ground penetrating radar; finite-difference time-domain method; perfectly matched layer; ricker wavelet

\section{INTRODUCTION}

At present, the topography, geological conditions and detection environment during tunnels and underground engineering construction are more and more complex. Water and mud bursting, massive landslides and other major engineering disasters occur with increasing frequency, and the casualties and economic losses are more and more serious ${ }^{[1]}$.

Therefore, in the construction of tunnels and underground engineering, adverse geological bodies must be detected in advance. Ground penetrating radar (GPR) is a fast and nondestructive detecting method with a good detecting effect in engineering. GPR uses the reflection and transmission of high frequency electromagnetic waves to determine the distribution patterns of the internal media ${ }^{[2,3]}$.

However, because of the diversity and complexity of the topography, geological conditions and detecting environment, the detection and interpretation of GPR is very difficult. Therefore, forward simulation models for the typical adverse geological bodies should be made and simulated, so that we can get the GPR response characteristics of these typical adverse geological bodies, consequently providing theoretical basis for the forecast and prevention in the practical project disasters.

Among numerous forward simulation methods for GPR detection, the finite-difference time-domain (FDTD) method

\author{
Lei Xu \\ Changjiang Engineering Geophysical Exploration Wuhan \\ CO. LTD \\ Wuhan, China
}

${ }^{[6,7]}$ is the most commonly used. In this paper, the theory of two-dimensional (2-D) FDTD method is introduced at first, then the forward simulation procedure of the FDTD program is introduced, and finally the effectiveness of the forward simulation method for GPR detection is proved by a numerical example.

\section{THEORY}

\section{A. Maxwell's Equations}

The propagation of electromagnetic field in media obeys Maxwell's equations strictly. The FDTD method is a set of time domain equations obtained by differencing the Maxwell's equations. For 2-D GPR forward simulation, considering the transverse magnetic (TM) waves, the Maxwell's equations ${ }^{[4]}$ can be written as:

$$
\left\{\begin{array}{l}
\frac{\partial E_{z}}{\partial y}=-\mu \frac{\partial H_{x}}{\partial t}-\sigma_{m} H_{x} \\
\frac{\partial E_{z}}{\partial x}=\mu \frac{\partial H_{y}}{\partial t}+\sigma_{m} H_{y} \\
\frac{\partial H_{y}}{\partial x}-\frac{\partial H_{x}}{\partial y}=\varepsilon \frac{\partial E_{z}}{\partial t}+\sigma E_{z}
\end{array}\right.
$$

Where $E$ is the electric field intensity $(\mathrm{V} / \mathrm{m}), H$ is the magnetic field intensity $(\mathrm{A} / \mathrm{m}), \sigma$ is the conductivity $(\mathrm{S} / \mathrm{m})$, $\sigma_{m}$ is the magnetic conductivity $(\Omega / \mathrm{m}), \varepsilon$ is the permittivity $(\mathrm{F} / \mathrm{m}), \mu$ is the permeability $(\mathrm{H} / \mathrm{m})$.

When the media is non-magnetic, there is $\sigma_{m}=0$ and $\mu=0$, therefore, only the impacts of $\sigma$ and $\varepsilon$ should be considered. The 2-D TM waves Maxwell's equations contain only three variables, $H_{x}, H_{y}$ and $E_{z}$, which represent the $x, y$ direction magnetic field intensity and the $\mathrm{z}$ direction electric field intensity respectively.

\section{B. FDTD Method}

In the 2-D FDTD method, the space is divided into $a \times b$ grids ( $a, b$ are the long and wide mesh number). The smallest unit of the differential grid is Yee cell ${ }^{[4]}$. The 2-D Yee cell is shown in Fig. 1. 


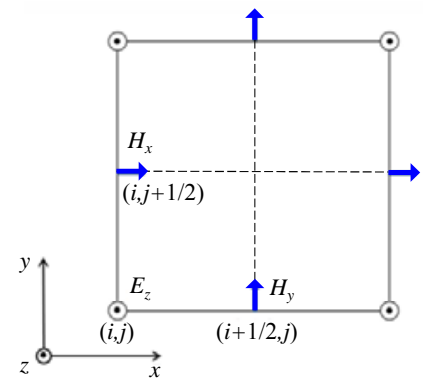

Fig. 1. The 2-D Yee cell

From Fig. 1 we can see that, each electric field component is surrounded by four magnetic field components, and each magnetic field component is surrounded by four electric field components. The electric field component and the magnetic field component are alternately sampled in the time sequence, which are different in time by half time step. Therefore, the FDTD equations ${ }^{[4,8]}$ of the 2-D TM waves can be obtained as follows:

$$
\left\{\begin{array}{l}
H_{x}^{n+1 / 2}(i, j+1 / 2)=C P \times H_{x}^{n-1 / 2}(i, j+1 / 2)- \\
C Q \times \frac{E_{z}^{n}(i, j+1)-E_{z}^{n}(i, j)}{\Delta y} \\
H_{y}^{n+1 / 2}(i+1 / 2, j)=C P \times H_{x}^{n-1 / 2}(i+1 / 2, j)+ \\
C Q \times \frac{E_{z}^{n}(i+1, j)-E_{z}^{n}(i, j)}{\Delta x} \\
E_{x}^{n+1}(i, j)=C A \times E_{x}^{n}(i, j)+ \\
C B \times\left[\frac{H_{y}^{n+1 / 2}(i+1 / 2, j)-H_{y}^{n+1 / 2}(i-1 / 2, j)}{\Delta y}-\right. \\
\left.\frac{H_{x}^{n+1 / 2}(i, j+1 / 2)-H_{x}^{n+1 / 2}(i, j-1 / 2)}{\Delta y}\right]
\end{array}\right.
$$

Where $\Delta x$ and $\Delta y$ are the $x$ and $y$ directions spatial step of the Yee cell, $\Delta t$ is the temporal step.

\section{Absorbing Boundary Conditions}

In order to simulate the propagation of electromagnetic waves in the open domain better, an electromagnetic waves absorbing layer needs to be arranged on the outer boundaries of the FDTD calculation area, so that the electromagnetic waves can enter the absorbing layer without reflection when reaching the boundaries, and the electromagnetic waves will decay quickly after entering the absorbing layer. In this paper, the selected electromagnetic waves absorbing layer is the perfectly matched layer (PML), and the 2-D PML absorbing boundary conditions ${ }^{[5]}$ are given by:

For $E_{z}$ :

$$
E_{z}(i, j)=E_{z x}(i, j)+E_{z y}(i, j)
$$

For $E_{z x}$ :

$$
\left\{\begin{array}{l}
E_{z x}^{n+1}(i, j)=C_{\text {ezxe }}(i, j) E_{z x}^{n}(i, j)+ \\
C_{\text {exxhy }}(i, j)\left[H_{y}^{n+1 / 2}(i, j)-H_{y}^{n+1 / 2}(i-1, j)\right] \\
C_{\text {ezxe }}(i, j)=\frac{2 \varepsilon_{0}-\Delta t \sigma_{x}(i, j)}{2 \varepsilon_{0}+\Delta t \sigma_{x}(i, j)} \\
C_{\text {ezxhy }}(i, j)=\frac{2 \Delta t}{\left[2 \varepsilon_{0}+\Delta t \sigma_{x}(i, j)\right] \Delta x}
\end{array}\right.
$$

For $E_{z y}$

$$
\left\{\begin{array}{l}
E_{\text {zy }}^{n+1}(i, j)=C_{\text {ezye }}(i, j) E_{z y}^{n}(i, j)+ \\
C_{\text {ezxhx }}(i, j)\left[H_{x}^{n+1 / 2}(i, j)-H_{x}^{n+1 / 2}(i, j-1)\right] \\
C_{\text {ezye }}(i, j)=\frac{2 \varepsilon_{0}-\Delta t \sigma_{y}(i, j)}{2 \varepsilon_{0}+\Delta t \sigma_{y}(i, j)} \\
C_{\text {ezxhx }}(i, j)=-\frac{2 \Delta t}{\left[2 \varepsilon_{0}+\Delta t \sigma_{y}(i, j)\right] \Delta y}
\end{array}\right.
$$

For $H_{x}$ :

$$
\left\{\begin{array}{l}
H_{x}^{n+1 / 2}(i, j)=C_{h x h}(i, j) H_{x}^{n-1 / 2}(i, j)+ \\
C_{h x e z}(i, j)\left[E_{z}^{n}(i, j+1)-E_{z}^{n}(i, j)\right] \\
C_{h x h}(i, j)=\frac{2 \mu_{0}-\Delta t \sigma_{m y}(i, j)}{2 \mu_{0}+\Delta t \sigma_{m y}(i, j)} \\
C_{h x e z}(i, j)=-\frac{2 \Delta t}{\left[2 \mu_{0}+\Delta t \sigma_{m y}(i, j)\right] \Delta y}
\end{array}\right.
$$

For $H_{y}$ :

$$
\left\{\begin{array}{l}
H_{y}^{n+1 / 2}(i, j)=C_{h y h}(i, j) H_{y}^{n-1 / 2}(i, j)+ \\
C_{\text {hyez }}(i, j)\left[E_{z}^{n}(i, j+1)-E_{z}^{n}(i, j)\right. \\
C_{\text {hyh }}(i, j)=\frac{2 \mu_{0}-\Delta t \sigma_{m x}(i, j)}{2 \mu_{0}+\Delta t \sigma_{m x}(i, j)} \\
C_{\text {hyez }}(i, j)=\frac{2 \Delta t}{\left[2 \mu_{0}+\Delta t \sigma_{m x}(i, j)\right] \Delta x}
\end{array}\right.
$$


Where $n$ is the order of the conductivity distribution, which has influence on boundary absorption effect.

\section{Reflection Coefficient}

The propagation of electromagnetic wave between media with different dielectric constants will cause reflection at the interface and the energy is decided by the reflection coefficient $\mathrm{R}^{[2]}$ :

$$
R=\frac{\sqrt{\varepsilon_{1}}-\sqrt{\varepsilon_{2}}}{\sqrt{\varepsilon_{1}}+\sqrt{\varepsilon_{2}}}
$$

Where $\varepsilon_{1}, \varepsilon_{2}$ are the relative dielectric constants of the media. When the value of $R$ is greater than 0 , the phase of the reflected waves are the same as the transmitting pulse; when the $R$ is less than 0 , the phase of the reflected wave is opposite to the transmitting pulse.

\section{E. 2-D forward Simulation Procedure}

The flow chart of the 2-D forward simulation procedure ${ }^{[6]}$ for GPR detection is shown in Fig. 2. The main steps are as follows:

- Make the 2-D forward simulation model, set the size, dielectric constants, conductivities and other parameters;

- Set the center frequency of the GPR antenna, the excitation source, the antenna positions, the time window and other parameters;

- Determine spatial step and temporal step, generate FDTD grids, and calculate the maximum time iterations;

- Initialize FDTD, PML parameters;

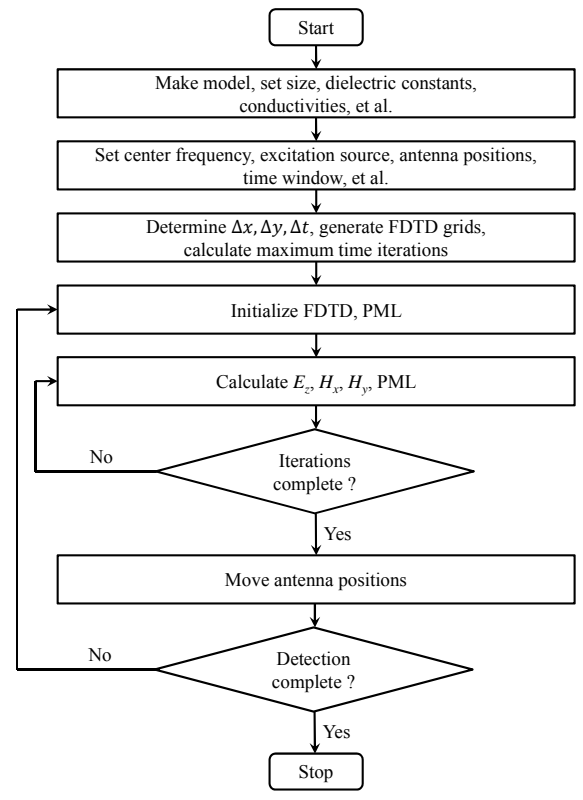

Fig. 2. Flowchart of 2-D forward simulation procedure
- Calculate $E_{z}, H_{x}$ and $H_{y}$ field at different time step, calculate the PML absorbing boundary conditions at the boundaries. Return to this step until the iterations are completed;

- Move antenna positions, go to step 4 until the detection is completed;

\section{EXAMPLES}

The 2-D forward simulation model is shown in Fig. 3. The model size is $20 \mathrm{~m} \times 10 \mathrm{~m}$ and it's divided into four parts. The upper medium has $\varepsilon_{r}=6, \sigma=1 \mathrm{mS} / \mathrm{m}$. The lower medium has $\varepsilon_{r}=12, \sigma=2 \mathrm{mS} / \mathrm{m}$. Within the upper medium there is a void having $\varepsilon_{r}=1, \sigma=0 \mathrm{mS} / \mathrm{m}$, and there is a water body having $\varepsilon_{r}=81, \sigma=10 \mathrm{mS} / \mathrm{m}$ in the lower medium.

The center frequency of the GPR antennas is $100 \mathrm{MHz}$. Antennas are located along the top interface of the model and moved from left to right with one spatial step at a time.

We use the ricker wavelet as the excitation source. The $100 \mathrm{MHz}$ Ricker wavelet is shown in Fig. 4 and takes the following form ${ }^{[9]}$ :

$$
I=-I_{0}\left[2 \pi^{2} f^{2}(t-1 / f)^{2}-1\right] e^{-\pi^{2} f^{2}(t-1 / f)^{2}}
$$

Where $I$ is the current, $I_{0}$ is the maximum current, $t$ is the time, $f$ is the center frequency of the GPR antennas.

For this model, we use $\Delta x=\Delta y=0.1 \mathrm{~m}$, so the model is divided into $200 \times 100$ grids. The time window is set to $250 \mathrm{~ns}$, and we use $\Delta t=0.2358 n$ s, so the iteration count is 1060 .

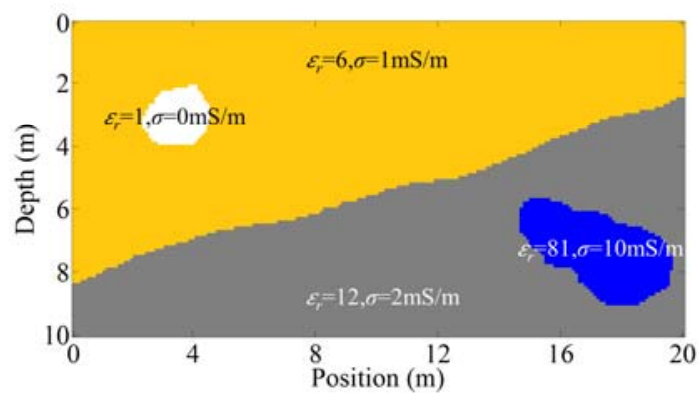

Fig. 3. 2-D forward simulation model

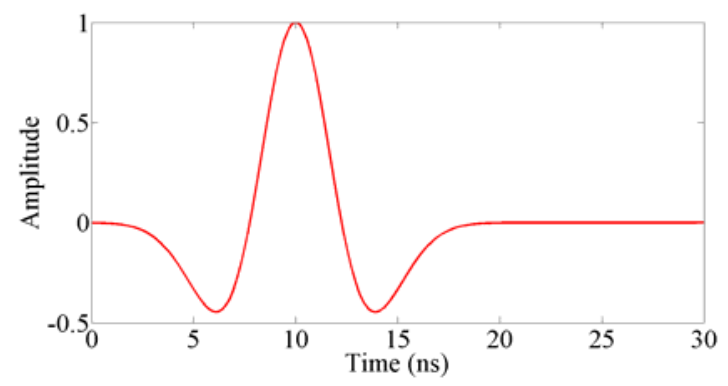

Fig. 4. Ricker wavelet 


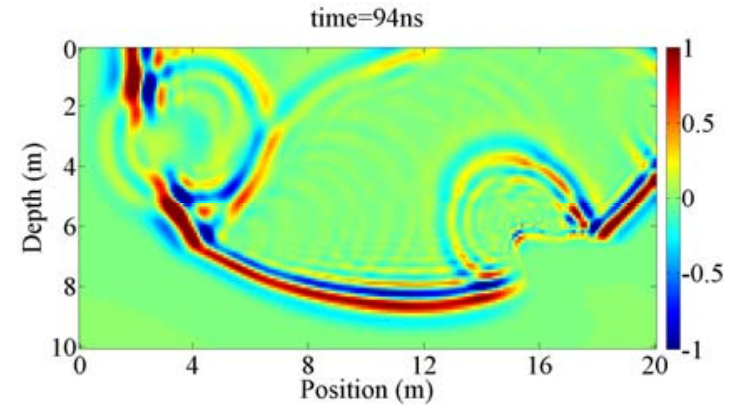

Fig. 5. Snapshot of $E_{z}$ field at position $=12 \mathrm{~m}$, time $=94 \mathrm{~ns}$

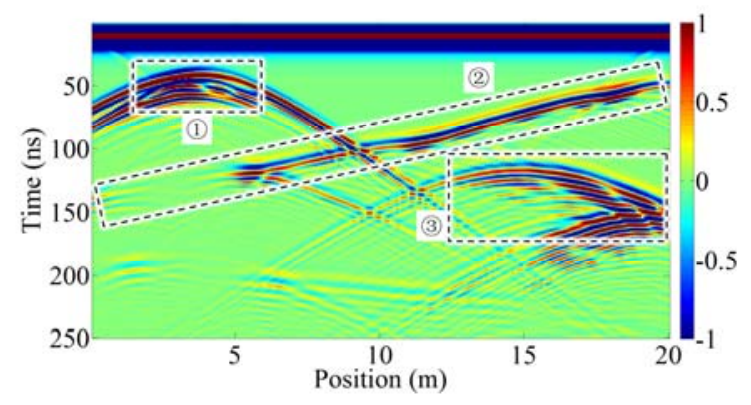

Fig. 6. 2-D forward simulation result

When the antennas are moved to $12 \mathrm{~m}$, the snapshot of $E_{z}$ field at time $=94 \mathrm{~ns}$ is shown in Fig. 5 . We can see that the absorption effect of the PML layer is great. No reflection occurs when the electromagnetic waves arrive at the boundaries of the model.

The result of the 2-D FDTD forward simulation is shown in Fig. 6. The hyperbolic shaped strong reflection can be seen in region (1) and (3), which can be inferred as adverse geological bodies. Layered reflection can be seen in region (2), which can be inferred as the interface of two different kinds of media. The phase of the reflected signal in region (1) is the same with as the transmitting pulse, and the phases of the reflected signals in region (2), (3) are opposite to the transmitting pulse. According to (9), we can infer that the dielectric constant of the upper medium is smaller than the lower medium, the dielectric constant of the adverse geological body in region (1) is smaller than the upper layer medium, and the dielectric constant of the adverse geological body in region (3) is greater than the lower layer medium.

\section{CONCLUSIONS}

This paper introduces the theory and procedure of the 2-D forward simulation method based on FDTD for GPR detection, which is proved to be effective through a numerical example: the position and shape of the adverse geological body can be inferred by analyzing the reflected signal from the FDTD simulation results; and the relationship between the relative dielectric constants of the adverse geological body and the surrounding medium can be found by analyzing the phase information of the reflected signal.

\section{REFERENCES}

[1] S. Li, The theory and method of geological prediction for the disaster source of water and mud inrush in tunnels. Beijing: China Science Publishing House, 2015.

[2] Z. Zeng, S. Liu, Z. Wang and J. Xue, The principle and application of ground penetrating radar. Beijing: Publishing House of Electronics Industry, 2010.

[3] S. Li, B. Liu, H. Sun, L. Nie, S. Zhong, et al., "State of art and trends of advanced geological prediction in tunnel construction," Chinese Journal of Rock Mechanics and Engineering, vol. 6, pp. 1090-1113, June 2014.

[4] K.S. Yee, "A perfectly matched layer for the absorption of electromagnetic waves," Journal of Computional Physics, vol. 2, pp. 185-200, January 1994.

[5] J.P. Berenger, "Numerical solution of initial boundary value problems involving maxwell's equations in isotropic media," Journal of Cliniacl Microbiology, vol. 3, pp. 302-307, March 1966.

[6] J. Irving, R. Knight, "Numerical modeling of ground-penetrating radar in 2-D using MATLAB," Computers \& Geosciences, vol. 9, pp. 12471258, September 2006.

[7] S. Gedney, Introduction to the Finite-Difference Time-Domain (FDTD) Method for Electromagnetics. California: Morgan \& Claypool Publishers, 2011.

[8] D. Ge, Finite-difference time-domain method for electromagnetic waves. Xian: XiDian University Press, 2011.

[9] C. Warren, A. Giannopoulos, Built-in waveforms, http://docs.gprmax. com/en/latest/plotting.html\#built-in-waveforms, accessed 20 December 2015 . 